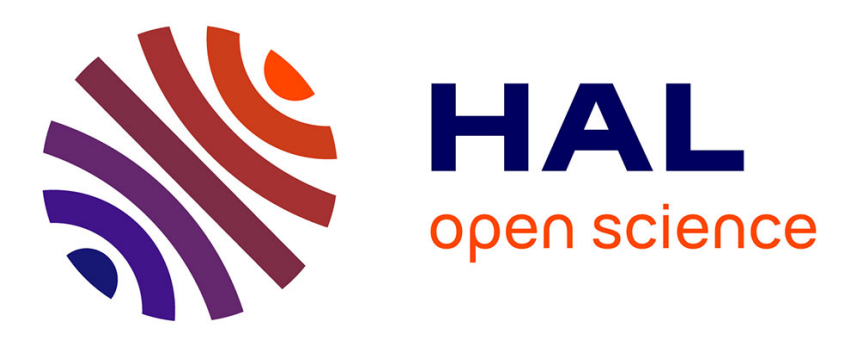

\title{
L'utilisation des Coléoptères subfossiles dans la reconstruction des paléo-environnements : l'exemple du port antique de Santa Pola (Espagne)
}

Pierre Moret, Carlos Martín Cantarino

\section{- To cite this version:}

Pierre Moret, Carlos Martín Cantarino. L'utilisation des Coléoptères subfossiles dans la reconstruction des paléo-environnements: l'exemple du port antique de Santa Pola (Espagne). Bulletin de la Société Entomologique de France, 1996, 101 (3), p. 225-229. hal-00723966

\section{HAL Id: hal-00723966 https://hal.science/hal-00723966}

Submitted on 16 Aug 2012

HAL is a multi-disciplinary open access archive for the deposit and dissemination of scientific research documents, whether they are published or not. The documents may come from teaching and research institutions in France or abroad, or from public or private research centers.
L'archive ouverte pluridisciplinaire HAL, est destinée au dépôt et à la diffusion de documents scientifiques de niveau recherche, publiés ou non, émanant des établissements d'enseignement et de recherche français ou étrangers, des laboratoires publics ou privés. 


\title{
L'utilisation des Coléoptères subfossiles dans la reconstruction des paléo-environnements : l'exemple du port antique de Santa Pola (Espagne)
}

\author{
par Pierre MORET* et Carlos MARTÍN-CANTARINO** \\ *63 chemin des Sept-Deniers, F - 31200 Toulouse \\ **Departamento de Ecología, Universidad de Alicante, Apartado de Correos 99, 03080 Alicante, España \\ Résumé. - Étude des Coléoptères subfossiles conservés dans un puits d'époque romaine (Ir $-I^{\mathrm{er}}$ siècles de \\ notre ère) à Santa Pola, sur le littoral sud-est de l'Espagne. \\ Summary. - Fossil Coleoptera were found in a Roman well used between the first and the fourth Century \\ AD at Santa Pola, on the south-east coast of Spain. Palaeoenvironmental implications are discussed. \\ Mots clés. - Coleoptera, archéo-entomologie, paléo-environnement, Espagne.
}

L'archéo-entomologie est une discipline encore embryonnaire en France et en Espagne, alors qu'elle connaît depuis plusieurs décennies de grands développements dans le nord de l'Europe, en particulier en Grande-Bretagne (BUCKLAND \& COOPE, 1991, SCOTT, 1994). Le but de cette note est, avant tout, de la faire mieux connaître, en attirant l'attention sur la richesse des informations paléo-environnementales que peut apporter l'étude des éléments d'entomofaune retrouvés par les archéologues dans certains types de dépôts archéologiques.

Les structures excavées alimentées en eau (puits, fosses, douves, latrines, etc.) constituent le milieu le plus favorable à la conservation des insectes subfossiles, à condition que les sédiments archéologiques n'aient jamais été exposés à l'air et à la dessication. Cette condition est moins souvent remplie dans les pays méditerranéens que dans l'Europe tempérée; c'est sans doute la raison pour laquelle l'étude que nous présentons est la première du genre qui ait été réalisée en Espagne.

\section{Le contexte archéologique}

Le site archéologique de La Picola, près du port moderne de Santa Pola (Alicante, Espagne), a fait l'objet de cinq campagnes de fouilles, de 1991 à 1995, par une équipe franco-espagnole (MORET et al., 1995). Les insectes qui motivent cette étude ont été recueillis dans un puits situé à proximité d'un complexe industriel romain de salaison de poisson. La mise en service du puits date de l'extrême fin du I ${ }^{\text {er }}$ siècle avant notre ère, et son comblement définitif se situe au $\mathrm{IV}^{\mathrm{e}}$ siècle de notre ère.

Ce puits atteint une profondeur de $2,46 \mathrm{~m}$ sous le sol actuel et de $0,16 \mathrm{~m}$ au-dessous du niveau de la mer, niveau qui est aussi celui de la nappe phréatique. Les dépôts antiques, au fond du puits, étaient recouverts sur une épaisseur de 1,63 m par un blocage de cailloux et de moellons. C'est dans les strates les plus profondes des sédiments archéologiques, audelà de deux mètres, que sont apparus en quantité importante des restes organiques d'origine végétale et animale. 
Aucun doute n'est possible quant à l'âge des insectes retrouvés à la base du puits. En effet, un insecte qui aurait pénétré dans le puits après son comblement (à partir de la fin du IV ${ }^{\mathrm{e}}$ siècle de notre ère) n'aurait pas pu traverser deux mètres de sédiments compacts, à moins d'être spécialement adapté au fouissement profond. Ce n'est le cas d'aucune des espèces étudiées. De plus, la texture relativement molle des téguments est caractéristique des insectes conservés très longtemps dans un milieu saturé d'eau.

\section{Matériel et méthode}

Cinq litres de sédiments provenant du niveau le plus profond du puits ont été tamisés puis triés sous la loupe binoculaire. Les insectes recueillis appartiennent à deux ordres seulement: Coleoptera et Hymenoptera. Ces derniers sont représentés par deux ou trois espèces de Formicidae qui n'ont pas été étudiées.

L'état de conservation des individus est généralement mauvais et se caractérise par la fragmentation des pièces chitineuses. Dans le meilleur des cas, on peut trouver un tibia et un fémur en connexion, ou une tête encore logée dans le pronotum, mais le plus souvent on n'a affaire qu'à des sclérites isolés. Ces sclérites, conservés à sec ou en alcool selon leur taille, n'ont pu être étudiés que par comparaison directe avec des exemplaires de collection, les clés de détermination étant souvent inutilisables quand on ne dispose que d'une seule pièce de l'exosquelette.

\section{Résultats}

On trouvera dans le tableau suivant la liste des taxa identifiés. Le nombre minimum d'individus observés est indiqué après chaque nom d'espèce (le nombre de fragments étant souvent supérieur).

$\begin{array}{lr}\text { Scarabaeidae: } & \\ \text { Scarabaeus sacer L. } & 5 \\ \text { Gymnopleurus geoffroyi (Fuessly) } & 2 \\ \text { Aphodiidae: } & 23 \\ \text { Platytomus tibialis (F.) } & 3 \\ \text { Aphodius (Calamosternus) granarius (L.) } & 1 \\ \text { Aphodius sp. } & 1 \\ \text { Aphodius sp. } & \\ \text { Dynastidae: } & 1 \\ \quad \text { Phyllognathus excavatus (Forster) }\end{array}$

\section{Discussion}

L'échantillon d'entomofaune conservé dans le puits romain de Santa Pola présente trois composantes principales.

1) Des espèces aptères, très actives au niveau du sol, qui sont tombées accidentellement dans le puits lors de leurs déplacements. Il s'agit des trois Tenebrionidae et du Chrysomelidae du genre Timarcha.

2) Des Scarabaeoidea coprophages et/ou saprophages (Scarabaeidae et Aphodiidae, six espèces) dont la présence dans le puits peut s'expliquer de deux façons. Soit ils ont été attirés par des excréments ou des matières en décomposition qui se trouvaient à proximité du puits, et y sont tombés accidentellement au cours de leur approche. Soit ils ont été attirés par des matières pourrissantes jetées dans le puits lui-même, à un moment où celui-ci avait cessé de servir à puiser l'eau. C'est vraisemblablement le cas de Platytomus tibialis, de loin l'espèce la plus abondante ( $48 \%$ du total des individus déterminés), dont la présence doit être mise en relation avec les débris végétaux en décomposition qui ont été retrouvés au fond du puits. Cette hypothèse est également 
envisageable pour Scarabaeus sacer. En effet, l'un de nous (C.M.) a observé, dans le même secteur côtier, de nombreux individus d'une autre espèce du genre, Scarabaeus semipunctıtus F., piégés dans un fossé où croupissait de l'eau usée.

3) Une espèce d'origine anthropique: le Curculionide Sitophilus granarius, ravageur bien connu des grains entreposés. On a retrouvé dans le puits plusieurs tessons de grandes jarres qui servaient à stocker des céréales; le charançon les accompagnait probablement. Cette espèce était déjà cosmopolite à l'époque romaine. Elle est attestée dès l'âge du bronze moyen, vers 1500-1300 avant notre ère, en Italie du Nord (FASANI, 1976), et dès le VII siècle, à l'aube de l'âge du fer, dans la province espagnole de Teruel, parmi des grains carbonisés (COMPTE \& PERALES, 1984). Au début de notre ère, elle est répandue dans tout l'Empire romain, du Proche-Orient jusqu'en Grande-Bretagne (KISLEV, 1991: 128). Sa présence dans le sud-est de l'Espagne n'est donc pas une surprise.

Bien que les espèces identifiées soient, au total, peu nombreuses, la majorité d'entre elles présente des spécialisations écologiques qui vont nous permettre de retracer avec précision la physionomie des environs du puits. Ces informations sont particulièrement précieuses dans le cas de Santa Pola, car des sondages effectués aux environs du site ont montré que la ligne de côte avait connu des déplacements importants au cours de l'Holocène. La plage actuelle se trouve à $400 \mathrm{~m}$ environ du site archéologique; au début du $I^{\text {er }}$ siècle de notre ère, une plage de sable fin bordait le site, à $25 \mathrm{~m}$ du puits; au III siècle, une plage de sable et de galets arrivait à $20 \mathrm{~m}$ du puits. L'environnement antique du site archéologique était donc bien différent de l'actuel.

Les deux espèces de Scarabaeidae, Scarabaeus sacer et Gymnopleurus geoffroyi, sont des espèces de milieu ouvert qui recherchent des terrains secs et non boisés, au sol bien drainé, avec une préférence, dans le cas de Scarabaeus sacer, pour les sols sablolimoneux meubles (LUMARET, 1990: 18). Il est difficile, en revanche, de déterminer la nature des excréments qui pouvaient les attirer dans les parages du puits romain. Les possibilités sont variées: vache, cheval, âne, brebis, homme. On notera l'absence de Scarabaeus semipunctatus $\mathrm{F}$., qui dans cette région montre une nette préférence pour les dunes du cordon littoral.

Chez les Aphodiidae, la présence d'Aphodius granarius est peu éclairante, car il s'agit d'une espèce ubiquiste, polyphage, qui tolère les environnements les plus variés (LumareT, 1990: 290). Platytomus tibialis est un témoin plus intéressant. Espèce littorale, aujourd'hui répandue dans la plus grande partie du bassin méditerranéen, sa biologie est mal connue. On sait cependant qu'elle affectionne la proximité des marécages littoraux et des étangs salés, où elle recherche les débris végétaux et le bois vermoulu (LUMARET, 1990: 316).

Les préférences édaphiques du Dynastidae Phyllognathus excavatus vont aux sols sablonneux (PAULIAN \& BARAUD, 1982: 381), mais il ne s'agit pas d'une exigence stricte.

Les Tenebrionidae représentés dans le puits sont des indicateurs écologiques particulièrement précis. Ce sont en effet des espèces aptères qui s'aventurent rarement hors de leur biotope. De plus, une étude écologique récente, consacrée aux Tenebrionidae psammophiles d'une dune située à El Saladar, à six kilomètres de Santa Pola (MARTín \& SEVA, 1989, 1990 et 1991), permet d'établir une comparaison très précise entre la faune dunaire actuelle et celle à laquelle appartenaient les espèces du puits romain.

Aucune des treize espèces de Tenebrionidae strictement psammophiles qui ont été observées dans la dune d'El Saladar (notamment Pimelia modesta Herbst, Erodius carinatus Solier, E. parvus Solier, Tentyria elongata Waltl, Pachychila frioli Solier) n'a été retrouvée dans le puits romain. En revanche, les espèces du puits sont les mêmes que 
l'on retrouve aujourd'hui sur des sols fermes, en arrière des cordons dunaires.

Le genre Tentyria est représenté aujourd'hui à Santa Pola par deux espèces: T. elongata Waltl, qui est un psammophile strict, et $T$. laevis Solier, très abondant dans les milieux arides à faible couvert végétal, les terrains vagues, les zones anthropisées. C'est cette dernière espèce qui a été retrouvée dans le puits romain.

Blaps gigas, présent dans le puits, est l'une des trois espèces de Blaps qui existent actuellement dans les environs de Santa Pola. Blaps lusitanica Herbst est propre aux milieux peu anthropisés, du type garrigue. $B$. sulcata brachyura Küst a une prédilection pour les formations dégradées, anthropisées, notamment les terrains vagues à proximité des agglomérations. $B$. gigas, qu'on trouve dans les mêmes milieux dégradés, est encore plus anthropophile, puisque on l'a souvent observé dans des bâtiments: caves, enclos, bergeries, etc. (ESPAÑOL, 1961, SAULEDA, 1977).

Scaurus punctatus est également une espèce rudérale, commune dans la province d'Alicante près des lieux habités.

Les restes de Timarcha retrouvés dans le puits peuvent appartenir à $T$. espagnoli Bechyné ou à $T$. intermedia Herrich-Schäffer. Ces deux espèces ont des préférences écologiques très voisines: toutes deux recherchent des terrains secs, éventuellement anthropisés, comme les friches ou les terrains vagues (JolIVET \& PETITPIERRE, 1973).

Enfin, les Buprestidae sont représentés par Melanophila cuspidata, petite espèce xylophage de mœurs carbonicoles. La larve vit aux dépens des pins (Pinus halepensis) et des genévriers (Juniperus oxycedrus) incendiés; sa préférence va à des troncs encore debout qui viennent d'être brûlés. Plus rarement, elle attaque des essences feuillues touchées par le feu (SCHAEFER, 1949: 223-226; 1984: 8). En Espagne, elle a été citée de la Catalogne, de Valence, de la Nouvelle-Castille et des Baléares (COBOS, 1986: 187). C'est le seul Coléoptère xylophage du puits; s'agissant d'une espèce ailée, capable de vols soutenus, il est impossible de se prononcer sur la distance à laquelle se trouvait sa plante-hôte.

\section{Conclusions}

Les conclusions que l'on doit tirer de l'analyse qui précède sont très claires. Le faubourg du port romain auquel appartenait le puits s'inscrivait dans un environnement semi-aride, fortement anthropisé, avec un couvert végétal faible. La proximité d'un étang salé, voire d'une saline, indispensable à l'époque romaine pour la fabrication des conserves de poisson, peut expliquer la présence de Platytomus tibialis.

Malgré la position littorale du site antique - les conserveries de poisson étaient bâties à quelques mètres de la mer -, on constate l'absence des espèces psammophiles qui sont si communes aujourd'hui sur la côte aux environs de Santa Pola. La plage, très étroite, devait donc s'arrêter brusquement contre une petite élévation de terrain, au sol sec et ferme, qui ne fut jamais envahie par le sable. Ces conditions favorables à limplantation humaine expliquent la forte anthropisation du milieu, bien reflétée par des espèces telles que Blaps gigas et Scaurus punctatus.

REMERCIEMENTs. - Yves Cambefort et Claude Girard, du Muséum National d'Histoire Naturelle, ont bien voulu nous prêter leur secours dans l'identification de Gymnopleurus geoffroyi et de Scaurus punctatus. Nous tenons aussi à remercier J.-P. Lumaret, pour ses précisions sur la biologie de Scarabaeus sacer, et P.C. Buckland, pour ses informations concernant les recherches archéo-entomologiques en Grande-Bretagne. 


\section{AUTEURS CITÉS}

BuCKLAND P.C. \& CoOPE G.R., 1991. - A Bibliography and Literature Review of Quaternary Entomology. University of Sheffieid, Department of Archaeology and Prehistory, $86 \mathrm{pp.}$

Cobos A., 1986. - Fauna ibérica de los Coleópteros Buprestidae. Madrid, C.S.I.C.

Compte A. \& Perales J., 1984. - Estudios de insectos coleópteros datados en el inicio de la iberización y pertenecientes al poblado de Siriguarach (Alcañiz, Teruel). Kalathos, 3-4 : 121-137.

EsPañol F., 1961. - Los Blaps de la Península Ibérica. Eos, 37 (4) : 399-414.

FASANI L., 1976. - Presenza di Sitophilus granarius (Linnaeus, 1758) (Coleoptera Curculionidae Calandrinae) in depositi dell'età del bronzo dell'Italia settentrionale, Tesi di Laurea di Scienze Naturali, Univ. Ferrara.

Jolivet P. \& PetitPierre E., 1973. - Plantes-hôtes connues des Timarcha Latreille (Col. Chrysomelidae). Bulletin de la Société entomologique de France, 78 : 9-24.

KISLEV M.E., 1991. - Archaeobotany and storage archaeoentomology. In Renfrew J.M. (ed.), New Light on Early Farming. Recent Developments in Palaeoethnobotany, Edinburgh University Press : 121-136.

LuMARET J.-P., 1990. - Atlas des Coléoptères Scarabéides Laparosticti de France. Paris, Muséum National d'Histoire Naturelle, Inventaires de faune et de flore, 1, $419 \mathrm{pp}$.

Martín-Cantarino C. \& Seva Román E., 1989. - Algunos aspectos de la coleopterofauna de las dunas costeras alicantinas (SE España). International Congress of Coleopterology, Barcelona, Abstracts: 117.

1990. - Ciclos de actividad y distribuciones espaciales en una comunidad de Coleoptera Tenebrionidae de las dunas costeras alicantinas (SE España). Mediterránea, Serie de Estudios Biológicos, 12: 91-112.

- 1991. - Morphological indices and resource partitioning in a guild of Coleoptera Tenebrionidae at the coastal sand-dunes of Alicante (SE Spain). In Zunino M., Bellés X., Blas M. (ed.), Advances in Coleopterology, Asociación Europea de Coleopterología, Barcelona : 211-222.

Moret P., Puigcerver A., Rouillard P., Sánchez M.J. \& Sillières P., 1995. - The Fortified Settlement of La Picola (Santa Pola, Alicante) and the Greek Influence in South-east Spain. In Cunliffe B. \& Keay S. (ed.), Social Complexity and the Development of Towns in Iberia, London, Proceedings of the British Academy, 86: 109-125.

PaUlian R. \& Baraud J., 1982. - Lucanoidea et Scarabaeoidea. Faune des Coléoptères de France, II, Paris, Lechevalier, $477 \mathrm{pp}$.

SAUlEDA N., 1977. - Contribución al conocimiento de la fauna halófila y psammófila de la Provincia de Alicante (Coleoptera y Heteroptera), su relación con la vegetación y creación y explotación de un banco de datos faunístico. Thèse de doctorat dactylographiée, Universidad de Valencia.

SCHAEFER L., 1949. - Les Buprestides de France. Supplément à Miscellanea Entomologica, Paris, $511 \mathrm{pp}$.

- 1984. - Les buprestes de France. Mise à jour 1983. Miscellanea Entomologica, 50 (1) : 1-15.

SCOTT A.E., 1994. - Quaternary Insects and Their Environments. Smithsonian Institution Press, 256 pp. 